\title{
Protective Effect of Polydatin Against Burn-Induced Lung Injury in Rats
}

\author{
Tao Li MSc, Shumin Cai MSc, Zhenhua Zeng MD, Junli Zhang MD, Youguang Gao MD, \\ Xingmin Wang MD, and Zhongqing Chen MD
}

\begin{abstract}
INTRODUCTION: Polydatin (PD) has anti-inflammatory and anti-apoptotic effects in ischemicreperfusion injury. Moreover, inflammatory responses and apoptosis play a role in the development of burn-induced lung injuries. Based on these findings, in this study we investigated the hypothesis that PD can ameliorate lung injury induced by extensive burns via reduction of inflammation and apoptosis. METHODS: Rats were subjected to $30 \%$ total body surface area burn injury followed by resuscitation. The treatment group received $45 \mathrm{mg} / \mathrm{kg} \mathrm{PD}$, and the burn group received the same amount of normal saline solution. No burn injury was inflicted in the sham group. Microvascular permeability, interstitial edema, neutrophil recruitment, and histopathological changes were detected by measuring Evans blue concentration, wet-to-dry lung weight ratio (W/D), myeloperoxidase (MPO) activity, and hematoxylin and eosin staining, respectively. To investigate the mechanism of action of PD, enzyme-linked immunosorbent assay, cell counting, terminal deoxyribonucleotidyl transferase-mediated deoxyuridine 5-triphosphate-digoxigenin nick end labeling (TUNEL) staining, fluorometric assay, and Western blot were used for assessing levels of inflammatory cytokines (tumor necrosis factor alpha, interleukin [IL]-1 $\beta$, and IL-6), total number of cells, and concentration of polymorphonuclear leukocytes (PMNs) in bronchoalveolar lavage fluid (BALF), the number of apoptotic cells, caspase-3 activity, and apoptosis-related proteins including Bax and Bcl-xl, respectively. RESULTS: Burn-injury rats exhibited significant lung injury characterized by the deterioration of histopathological characteristics, pulmonary microvascular hyperpermeability, and a high W/D, which were attenuated by PD $(P=.007$ for permeability, $P=.004$ for W/D). PD inhibited the burn-induced inflammatory response, as evidenced by the down-regulation of lung MPO activity $(P=.008)$, total number of cells, PMN concentration in BALF, and the local and systemic levels of the pro-inflammatory cytokines examined. Moreover, PD treatment dramatically prevented burn-induced pulmonary cell apoptosis in lungs, as reflected by the decrease in the number of TUNEL-positive cells $(P=.002)$ and changes in Bax, Bcl-xl, and caspase-3 activity $(P=.03)$. CONCLUSIONS: PD ameliorates burn-induced lung injury via its anti-inflammatory and anti-apoptotic effects, and PD treatment may therefore serve as a potential therapeutic target for the treatment of critical burn injuries. Key words: polydatin; extensive burn; lung injury; permeability; apoptosis; inflammation. [Respir Care 2014;59(9):1412-1421. () 2014 Daedalus Enterprises]
\end{abstract}

\section{Introduction}

Burn injury is a common trauma, and the lungs are some of the most vulnerable distant organs that are affected by

Drs Li, Cai, Zeng, Zhang, Gao, and Chen are affiliated with the Department of Critical Care Medicine, Nanfang Hospital, Southern Medical University, Guangzhou, People's Republic of China. Dr Zeng is affiliated extensive burns. Acute lung injury (ALI) is associated with high morbidity and mortality in burn patients with extensive skin burns, and it is clinically characterized by hypoxemia, noncardiogenic pulmonary edema, and rapid 
respiratory failure. ${ }^{1-7}$ The pathophysiology of ALI involves highly complicated mechanisms, and recent studies ${ }^{3,8-16}$ have shown that systemic inflammatory response, apoptosis, and endothelial cell dysfunction are key to this process.

The role of inflammatory mediators in ALI is welldescribed. Many inflammatory factors enter the circulation after local burn injury and cause a severe systemic inflammatory response. An excessive inflammatory response constitutes the earliest stages in the development of lung injury after burn injury. Increased levels of local and systemic inflammatory mediators, such as tumor necrosis factor alpha (TNF- $\alpha$ ) and interleukin (IL)- $1 \beta$, IL-6, and activated leukocytes, lead to systemic inflammatory response syndrome (SIRS). In addition, inflammatory mediators recruit neutrophils into the alveolar-capillary wall, adhere to the endothelium, release various proteases, and infiltrate into the injury site. These processes may result in damage to the alveolar-capillary membrane and lead to increased permeability of the endothelium in patients with lung injury. ${ }^{17,18}$ Vascular hyperpermeability enhances the infiltration of inflammatory cells, notably polymorphonuclear leukocytes (PMNs), and subsequently results in cell apoptosis and interstitial edema. . $3,5,19,20,21$ In recent years, increasing evidence has suggested that pulmonary cell apoptosis may also play an important role in the pathophysiology of lung injury. ${ }^{16,22-24}$ All these processes subsequently lead to pulmonary damage.

Polydatin (PD), a monocrystalline drug that can be isolated from the traditional Chinese herb Polygonum cuspidatum, is used mainly in the treatment of shock, burn, and ischemia-reperfusion injury. ${ }^{25-28}$ The effect of PD on acute severe shock and burn injury has been studied at our laboratory for many years, and we, so far, have not discovered any side effect of PD. The Sino Food and Drug Administration has approved of a clinical trial investigating the effect of PD on hypotension after hemorrhagic shock, and the trial has now entered stage II. Moreover, the in vitro and in vivo influence of PD on inflammatory processes

Department of Clinical Laboratory, Maternal and Child Health Hospital of Liuzhou, Liu Zhou, People's Republic of China. Dr Gao is affiliated with the Department of Anesthesiology, The First Affiliated Hospital of Fujian Medical University, Fuzhou, Fujian Province, People's Republic of China.

The authors have disclosed no conflicts of interest.

Drs Li and Cai are co-first authors of this paper.

Correspondence: Zhongqing Chen MD, Department of Critical Care Medicine, Nanfang Hospital, Southern Medical University, 1838 Guangzhou Avenue North, Guangzhou 510515, People's Republic of China. E-mail: zhongqingchen.edu@gmail.com.

DOI: $10.4187 /$ respcare.02831

\section{QUICK LOOK}

\section{Current knowledge}

Polydatin is a monocrystalline drug isolated from the traditional Chinese herb Polygonum cuspidatum, which is used in the treatment of shock, burn, and ischemiareperfusion injury.

\section{What this paper contributes to our knowledge}

In an animal model of burn injury, polydatin ameliorates burn-induced lung injury via anti-inflammatory and anti-apoptotic effects. The role of polydatin as a potential therapeutic target for the treatment of critical burn injuries requires further study.

and apoptosis has been demonstrated efficiently in several models. $25,26,29-33$

Since inflammatory response and apoptosis play key roles in the pathogenesis of burn-induced lung injury and PD has potential anti-inflammatory and anti-apoptosis effects, we hypothesized that PD may provide protection against burn-induced lung injury.

\section{Methods}

\section{Experimental Animals}

The procedures used in this study and the handling of study animals adhered to the National Institutes of Health guidelines on the use of experimental animals. The experimental protocol was approved by the Committee on Research Animal Use of South Medical University. Male Sprague-Dawley rats weighing 180-220 g were purchased from the Experimental Animal Center at South Medical University and allowed to acclimatize for a week before being used. Animals had ad libitum access to chow and water.

\section{Experimental Groups and Animal Model}

As shown in Figure 1, animals were randomly divided into the following 3 groups (1): sham burn $(n=20)$; (2) burn + normal saline solution (NS) $(n=20)$; and (3) burn + PD $(n=20)$ (Table 1). All the rats were anesthetized with an intramuscular injection of sodium pentobarbital $(30 \mathrm{mg} / \mathrm{kg})$. Burn injury was inflicted based on a modified Walker burn model. ${ }^{34}$ Briefly, a dorsal area that equals $30 \%$ of the total body surface area was shaved. The rat was placed in a mold with an adjustable opening to expose the shaved area to $98^{\circ} \mathrm{C}$ water for $30 \mathrm{~s}$. Animals in the sham group were immersed in water at room temper- 


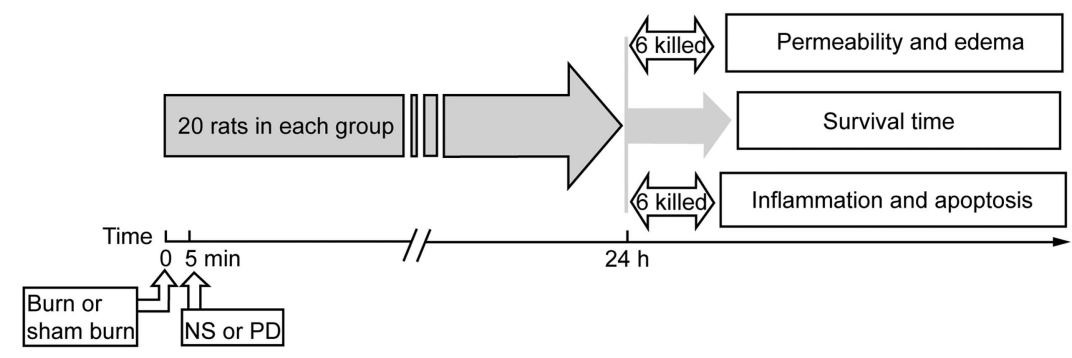

Fig. 1. Experiment sequence, groups, and subgroups. $N S=$ normal saline solution; $P D=$ polydatin.

Table 1. Burn Injury-Induced Changes in Lung Tissue and Serum (Chi-Square Test)

\begin{tabular}{|c|c|c|c|c|c|}
\hline Group & Sham burn & Burn + NS & Burn + PD & Sham vs Burn + NS & Burn + NS vs Burn + PD \\
\hline $\begin{array}{c}\mathrm{EB}(\mu \mathrm{g} / 100 \mathrm{mg} \\
\text { dry tissue })\end{array}$ & $11.84 \pm 1.88$ & $24 \pm 5.22$ & $17.66 \pm 2.99$ & $<.001$ & .007 \\
\hline $\mathrm{W} / \mathrm{D}$ & $3.62 \pm 0.33$ & $4.83 \pm 0.38$ & $4.00 \pm 0.25$ & $<.001$ & .004 \\
\hline \multicolumn{6}{|l|}{ Systemic (pg/mL) } \\
\hline TNF- $\alpha$ & $19 \pm 12.3$ & $432 \pm 122.7$ & $229 \pm 66.8$ & $<.001$ & $<.001$ \\
\hline IL-1 $\beta$ & $33 \pm 20.8$ & $310 \pm 102.8$ & $194 \pm 60.8$ & $<.001$ & .004 \\
\hline IL-6 & $26 \pm 12.5$ & $230 \pm 88.5$ & $150 \pm 48.7$ & $<.001$ & .02 \\
\hline \multicolumn{6}{|l|}{ Local ( $\mu \mathrm{g} / \mathrm{mg}$ tissue) } \\
\hline TNF- $\alpha$ & $24 \pm 7.4$ & $332 \pm 68.5$ & $95 \pm 22.6$ & $<.001$ & .001 \\
\hline $\mathrm{IL}-1 \beta$ & $13 \pm 4.2$ & $172 \pm 73.8$ & $85 \pm 25.4$ & $<.001$ & .01 \\
\hline IL-6 & $31 \pm 11.3$ & $188 \pm 65.2$ & $120 \pm 27.0$ & $<.001$ & .033 \\
\hline Total cells $\left(10^{5} / \mathrm{mL}\right)$ & $0.49 \pm 0.2$ & $9.53 \pm 2.11$ & $5.90 \pm 1.97$ & $<.001$ & .002 \\
\hline PMNs $\left(10^{5} / \mathrm{mL}\right)$ & $0.24 \pm 0.16$ & $6.42 \pm 2.31$ & $3.79 \pm 1.73$ & $<.001$ & .02 \\
\hline $\begin{array}{l}\text { TUNEL-positive } \\
\text { cells/field }\end{array}$ & $1.7 \pm 1.2$ & $39.5 \pm 12.7$ & $18.7 \pm 10.6$ & $<.001$ & .002 \\
\hline $\begin{array}{l}\text { Data are mean } \pm \text { SD. } \\
\text { NS = normal saline solution } \\
\mathrm{EB}=\text { Evans blue } \\
\mathrm{PD}=\text { polydatin } \\
\mathrm{W} / \mathrm{D}=\text { wet-to-dry lung weig } \\
\mathrm{TNF}=\text { tumor necrosis facto } \\
\mathrm{IL}=\text { interleukin } \\
\mathrm{PMN}=\text { polymorphonuclear } \\
\text { TUNEL = terminal deoxyrib }\end{array}$ & 1 transferase-med & uridine 5 -triphos & igenin nick end 1 & & \\
\hline
\end{tabular}

ature. Then, the rats were resuscitated using an intraperitoneal injection of Ringer's lactate solution, with the volume calculated using the Parkland formula $(4 \mathrm{~mL} / \mathrm{kg}$ per percentage of the burn). In the PD treatment group, rats were given $45 \mathrm{mg} / \mathrm{kg}$ PD (Neptunus Co., Shenzhen, People's Republic of China) by femoral vein injection at $5 \mathrm{~min}$ after the burn injury. The dose was based on the authors' previous research data. Animals in the sham burn group did not receive any additional treatment. After the burn injury was inflicted and treatment was given, each of the 3 groups was divided into 3 subgroups for survival time investigation $(n=8)$, permeability measurement $(n=6)$, and inflammation and apoptosis assay $(n=6)$. The rats in the survival time subgroup were kept in a temperaturecontrolled room and were given water ad libitum after the burn. The rest were killed at $24 \mathrm{~h}$ postburn for measurements.

\section{Histologic Examination}

Lungs were harvested for observing morphologic alterations at $24 \mathrm{~h}$ after burn or sham burn. The right middle lobes of the lungs were fixed with $10 \%$ formalin, embedded in paraffin, and sectioned to $4-\mu \mathrm{m}$ thickness. After deparaffinization and rehydration, the sections were stained with hematoxylin and eosin. The pathological sections were observed in a blinded fashion.

\section{Lung Microvascular Permeability Assay}

For the permeability assay, vascular protein leakage was measured using the Evans blue (EB) technique. ${ }^{35,36}$ After anesthetization, EB (20 mg/kg weight; Sigma, St. Louis, Missouri) was injected intravenously through the femoral 
vein. Thirty minutes after dye injection, the animals were killed, and a midline thoracotomy was performed. Then the superior and inferior vena cavae were ligated, the aorta was transected, and $20 \mathrm{~mL}$ of NS was injected into the right ventricle at a pressure of $20 \mathrm{~cm} \mathrm{H}_{2} \mathrm{O}$ to wash out the pulmonary intravascular content. A sample of lung tissue was weighed, immersed in $\mathrm{N}, \mathrm{N}$-dimethylformamide (Sigma), and homogenized. The homogenate was incubated at room temperature for $48 \mathrm{~h}$. Eluted EB was measured at $620 \mathrm{~nm}$ using an automatic microplate reader (SpectraMax M5; Molecular Devices, Sunnyvale, California), and the amount was expressed as micrograms per $100 \mathrm{mg}$ dry tissue.

\section{Wet Lung/Dry Lung Weight Ratio}

The harvested wet lung was weighed and then placed in an oven for $48 \mathrm{~h}$ at $80^{\circ} \mathrm{C}$ and then weighed again when it was dry. The wet-to-dry lung weight ratio (W/D) was calculated.

\section{Cell Counts in Bronchoalveolar Lavage Fluid}

Rats were killed, a median sternotomy was performed, and the trachea was isolated by blunt dissection; then, a suitable small-caliber tube was inserted into the airway and secured. Phosphate-buffered saline solution $(\mathrm{pH}$ 7.2) was infused slowly into the lungs, and bronchoalveolar lavage fluid (BALF) was withdrawn via the tube. The fluid recovery rate was $>90 \%$. Lavage samples were centrifuged at $1,500 \mathrm{~g}$ for $10 \mathrm{~min}$ at $4^{\circ} \mathrm{C}$. The sedimented cells were resuspended in phosphate-buffered saline solution and subjected to cell counting. The slides were visualized using Wright-Giemsa staining (Sigma), and PMNs were counted in a double-blind fashion.

\section{Measurement of Inflammatory Mediators}

The concentrations of TNF- $\alpha$, IL- $1 \beta$, and IL- 6 in the lung or serum were measured using commercial enzymelinked immunosorbent assay kits (R\&D Systems, Minneapolis, Minnesota). The results were expressed as micrograms per milligram tissue or pictograms per milliliter serum.

\section{Myeloperoxidase Activity Assay}

Lungs were harvested, rinsed, homogenized, and centrifuged. Supernatants were collected and subjected to enzyme-linked immunosorbent assay for determination of myeloperoxidase (MPO) activity using the MPO activity colorimetric assay kit (Biovision, Zurich, Switzerland).
Terminal Deoxyribonucleotidyl Transferase-Mediated Deoxyuridine 5-Triphosphate-Digoxigenin Nick End Labeling Staining

Lung histopathological slides were dewaxed and incubated with proteinase K. Slides were stained using a terminal deoxyribonucleotidyl transferase-mediated deoxyuridine 5-triphosphate-digoxigenin nick end labeling (TUNEL) kit (Biovision), counterstained with Hoechst 33258 stain and examined under a fluorescence microscope. Apoptotic cells appeared fluorescent green and were counted per 10 visual fields at a $200 \times$ magnification.

\section{Caspase-3 Activity}

Lung homogenates were prepared, and the caspase-3 activity was measured using the caspase-3/CPP32 fluorometric assay kit (Biovision) in accordance with the manufacturer's instructions.

\section{Western Blot Analysis for Bcl-xl-Bax}

The lung tissues were homogenized and analyzed for Bcl-xl-Bax by Western blotting. Protein concentrations were determined using the bicinchoninic acid method. An equal amount of protein was loaded onto $10 \%$ sodium dodecyl sulfate polyacrylamide gels for electrophoresis. After electrophoresis, proteins were electroblotted onto polyvinylidene fluoride membranes and blotted with primary antibodies against Bcl-xl-Bax (Abcam, Cambridge, United Kingdom) and $\beta$-actin (Tianjin Sungene Biotech Co., Ltd., Tianjin, People's Republic of China). Membranes were then incubated with the horseradish peroxidase-tagged secondary antibody (Earth, UK), and protein expression was detected using an enhanced chemiluminescence reagent.

\section{Statistical Analysis}

All variables are presented as the mean \pm SD. Differences between groups were determined using one-way analysis of variance with the least significant difference multiple-comparison test and Student $t$ test when appropriate. Values were considered to be significant at $P<.05$, and $n$ represents the number of animals.

\section{Results}

\section{PD Treatment Attenuated Burn-Induced Lung Injury}

Lung tissue of rats in the burn + NS group showed accumulation of a large number of neutrophils in the intraalveolar and interalveolar space, a thickened alveolar wall, 


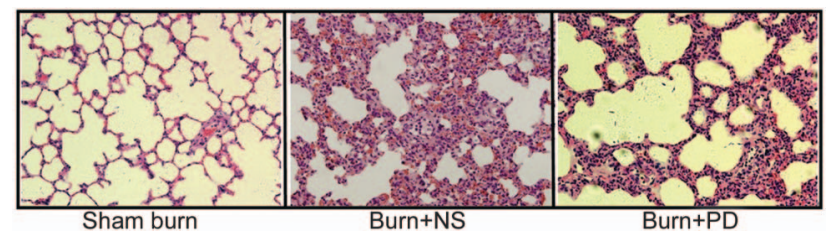

Fig. 2. Effect of polydatin (PD) on lung histopathology changes in burn-injury rats. Lungs harvested from each group at $24 \mathrm{~h}$ after burn injury were stained with hematoxylin and eosin, and examined under a light microscope. NS = normal saline solution; $\mathrm{PD}=$ polydatin

less alveolar space, interstitial congestion, and edema. PD treatment was found to markedly attenuate these signs of burn-induced lung injury (Fig. 2).

\section{PD Treatment Inhibited Burn-Induced Pulmonary Microvascular Hyperpermeability}

Burn injury results in a sustained increase in microvascular permeability, which directly results in pulmonary edema. Therefore, we analyzed microvascular permeability by measuring the EB concentration in lungs, as the EB concentration is proportional to microvascular permeability. EB concentration in the lung was markedly increased in postburn rats (sham group vs burn + NS group: $P<.01$ ). Treatment of PD significantly reduced the concentration of EB in the lung (burn + PD group vs burn + NS group: $P=.007)$; therefore, $\mathrm{PD}$ treatment inhibits microvascular hyperpermeability induced by burn injury (Fig. 3A, Table $1)$.

\section{PD Treatment Reduced Pulmonary Edema Induced by Burn Injury}

Pulmonary edema is one of the most characteristic pathologic changes in burn-induced lung injury. To determine whether PD exerts a protective effect on pulmonary edema, we compared the W/D among the 3 different treated groups. At $24 \mathrm{~h}$ after burn injury was induced the W/D in the burn-injury animals was significantly increased compared with that in the sham controls (sham group vs burn + NS group: $P<.01$; Fig. 3, Table. 1). Administration of PD markedly decreased the W/D compared with that in burn + NS rats (burn + PD group vs burn + NS group: $P=.004)$.

\section{PD Treatment Attenuated Local and Systemic Levels of Inflammatory Mediators Induced by Burn Injury}

Given the key role of inflammatory mediators in SIRS and ALI, we measured the lung tissue and serum concentrations of inflammatory mediators in the different groups. TNF- $\alpha$, IL- $1 \beta$, and IL- 6 levels in the lungs of the burn
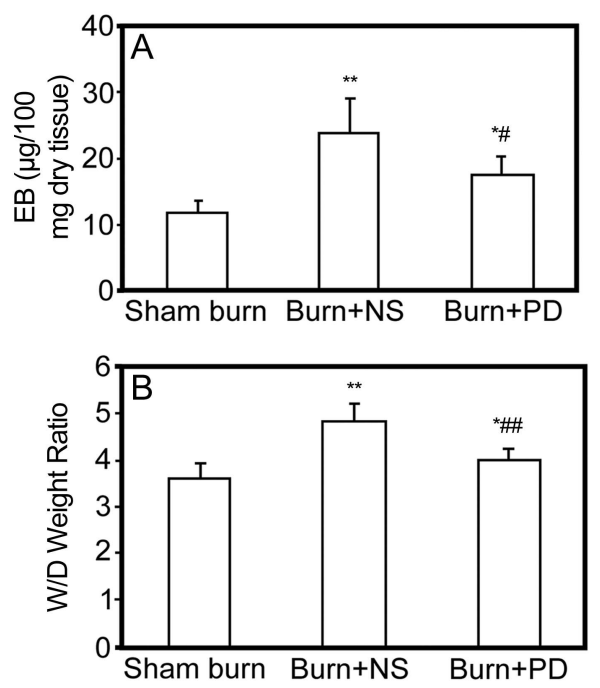

Fig. 3. Influence of polydatin (PD) on pulmonary microvascular permeability and edema. A: Evans blue (EB) was extracted from the lungs obtained from each group at $24 \mathrm{~h}$ postburn and measured. B: Wet-to-dry lung weight ratio (W/D). Both the EB and W/D values were increased in the burn + normal saline solution (NS) group, but were alleviated in the burn + PD group. Data are presented as the mean $\pm \mathrm{SD}\left(n=6\right.$ in each group). ${ }^{*} P=.05,{ }^{* *} P<.01$ vs the sham burn group; $\# P<.05, \# \#<.01$ vs the burn + NS group.

animals were dramatically higher than those in sham animals (sham group vs burn + NS group: $P<.01$ for all; Fig. 4A, Table 1), and these levels were significantly reduced in the PD treatment group (burn + PD group vs burn + NS group: $P<.001$ for TNF- $\alpha, P=.004$ for IL-1 $\beta, P=.01$ for IL-6). Similar to their levels in lung tissues, the TNF- $\alpha$, IL- $1 \beta$, and IL- 6 levels in the peripheral blood of burn animals were significantly higher than those in sham animals (sham group vs burn + NS group: $P<.01$ for all; Fig. 4B, Table 1). The increased levels of TNF- $\alpha$, IL- $1 \beta$, and IL- 6 were markedly attenuated by PD treatment (in burn + PD group vs burn + NS group: $P=.001, P=.01$, and $P=.033$, respectively).

\section{PD Treatment Reduced the Total Number of Cells and PMNs in the BALF}

A marked increase in the total number of cells and PMNs was noted in the BALF in burn-injury rats (sham group vs burn + NS group: $P<.01$ for both; Fig. 5A,B, Table 1), which were markedly reduced by PD treatment (burn + PD vs burn + NS group: $P=.002$ for total number of cells, and $P=.02$ for PMNs).

\section{PD Treatment Decreased Neutrophils Recruitment in the Lung}

Lung MPO activity, an indicator of neutrophil infiltration, was detected. As shown in Figure 5C, the postburn 

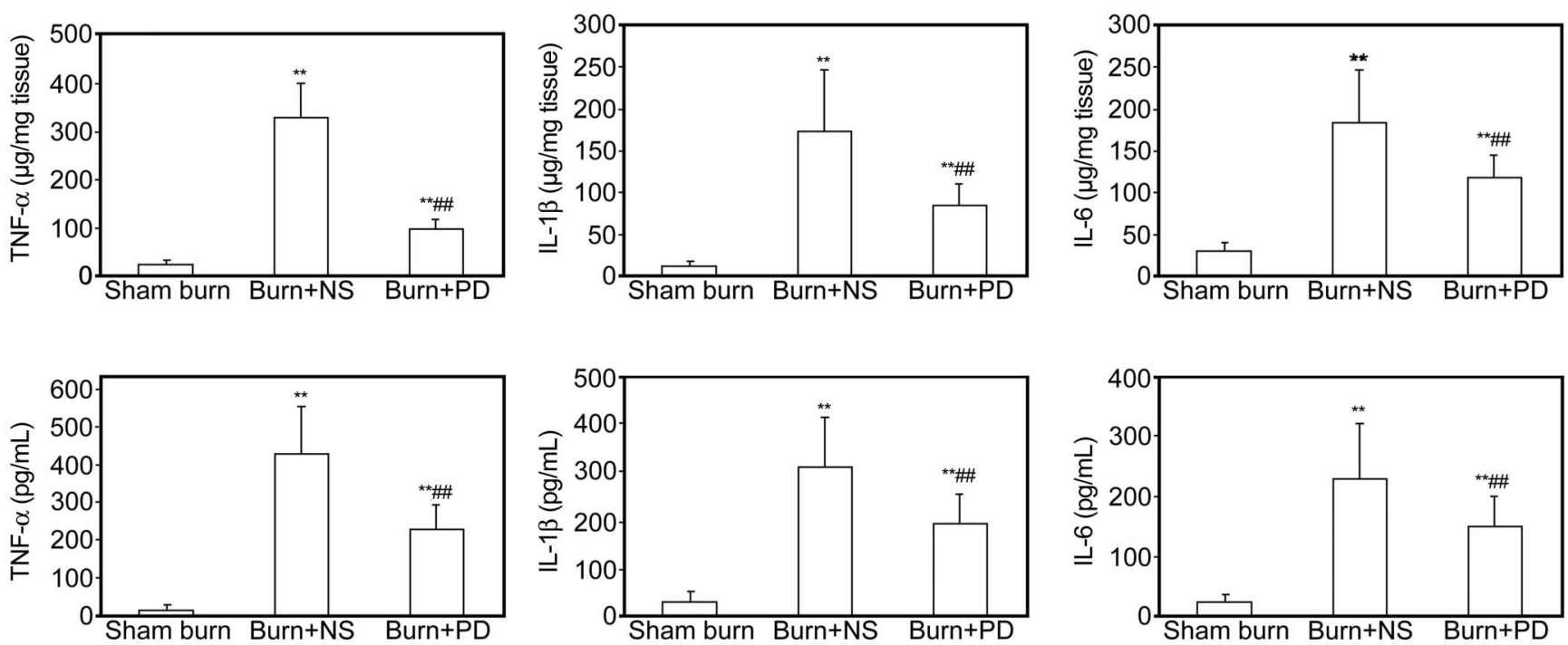

Fig. 4. Effect of polydatin (PD) on local and systemic inflammatory mediators after burn injury. (Top) The protein levels of tumor necrosis factor alpha (TNF- $\alpha$ ), interleukin (IL)-1 $\beta$, and IL- 6 in the lung were determined at $24 \mathrm{~h}$ postburn. (Bottom) The circulating levels of inflammatory mediators in the serum were measured at $24 \mathrm{~h}$ after burn. The pulmonary and serum levels of all 3 factors were elevated in the burn + NS group, but decreased in the burn + PD group. Data are presented as the mean \pm SD $\left(n=6\right.$ in each group). ${ }^{\star} P<.05$, ${ }^{\star \star} P<.01$, vs the sham burn group; \#P<.05, \#\#P<.01 vs the burn + normal saline solution (NS) group.
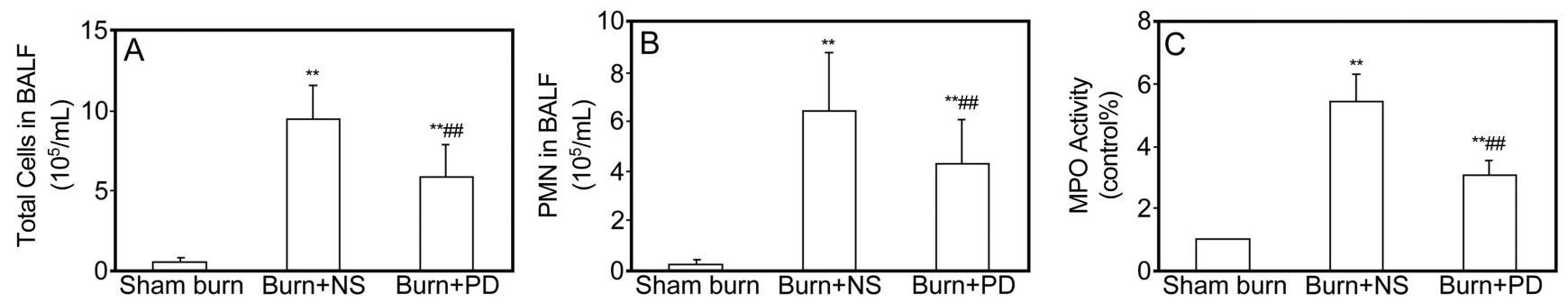

Fig. 5. Effect of polydatin (PD) on the total number of cells and polymorphonuclear leukocytes (PMNs) in the bronchoalveolar lavage fluid (BALF) and lung myeloperoxidase (MPO) activity. A: Total number of cells in BALF. B: PMNs in BALF. C: Lung MPO activity. The values of all 3 factors were increased in the burn + normal saline solution (NS) group, but were decreased in the burn + PD group. Data are presented as mean $\pm \mathrm{SD}\left(n=6\right.$ in each group). ${ }^{\star} P<.05,{ }^{\star \star} P<.01$ vs the sham burn group; \#P<.05, \#\#P<.01 vs the burn + NS group.

lung MPO activity dramatically increased (sham group vs burn + NS group: $P<.01$ ), and it was inhibited by PD treatment (burn $+\mathrm{NS}$ group vs burn + PD group: $P=.008$ )

\section{PD Treatment Prevented Lung Cell Apoptosis in Burn-Injury Rats}

To detect the effects of PD treatment on pulmonary cell apoptosis in burn-injury rats, TUNEL assay, a common method for detecting DNA fragmentation, was conducted on the lung tissues. The burn-injury animals showed a significant increase in apoptotic cells in comparison with the sham burn group (sham group vs burn + NS group: $P<.01)$; this was significantly reduced by PD treatment (burn + PD group vs burn + NS group: $P=.002$; Figs. 6, 7; Table 1).
Moreover, we investigated the effects of PD treatment on pulmonary cell apoptosis by detecting the level of Bclxl-Bax and caspase-3 activity. Overexpression of Bcl-xl ameliorates lung injury by inhibiting apoptotic pathways. Burn injury resulted in down-regulation of the Bcl-xl protein, and PD prevented this decrease. Expression of the pro-apoptotic protein Bax was up-regulated by burn injury and inhibited by PD treatment (Fig. 8). Activation of caspases plays a central role in the process of cellular apoptosis. We therefore measured caspase- 3 activity and found that it was significantly increased in the lungs of animals after burn injury, which was prevented by PD treatment (sham group vs burn + NS group: $P<.01$; burn + NS group vs burn + PD group: $P=.03$ ). These results indicate that burn injury induce the lung cell apo- 

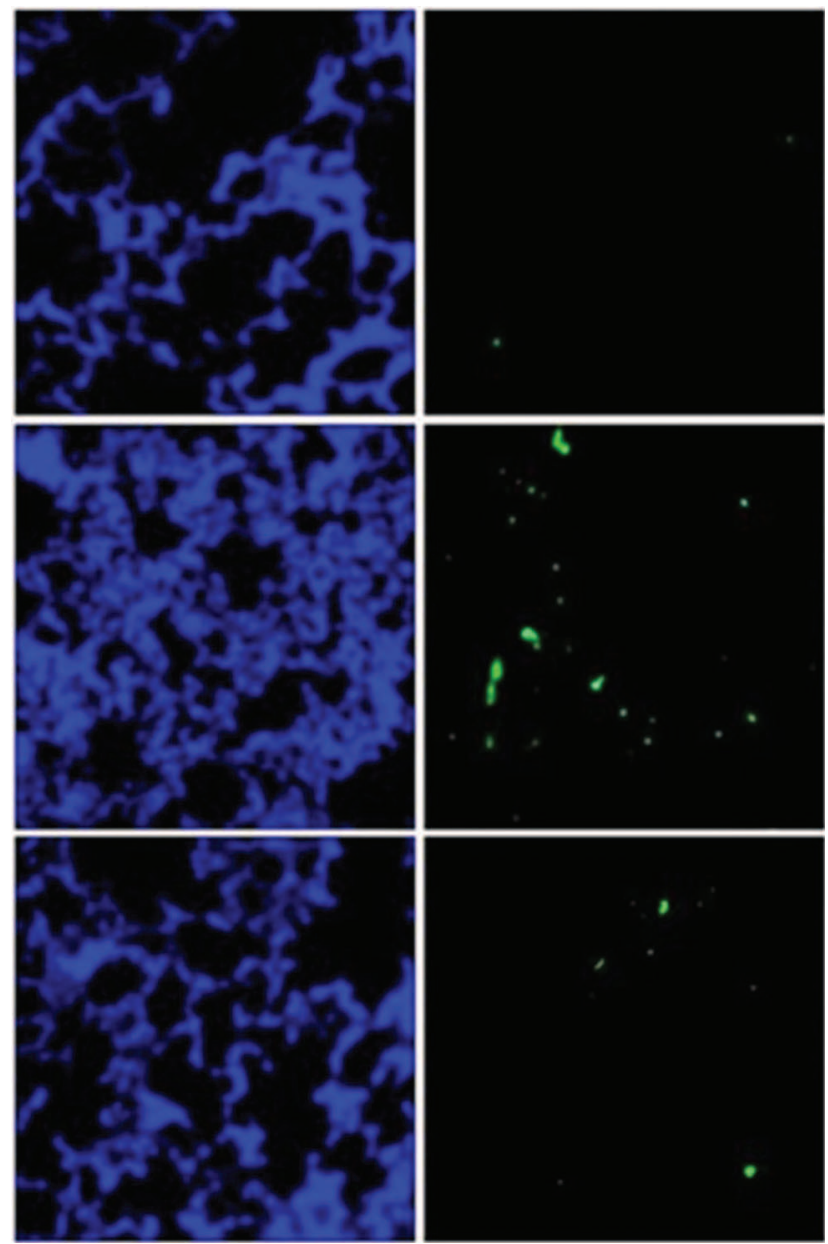

Fig. 6. Effects of polydatin on apoptosis in the lungs after burn injury. Terminal deoxyribonucleotidyl transferase-mediated deoxyuridine 5-triphosphate-digoxigenin nick end labeling staining images shown at $200 \times$ magnification.

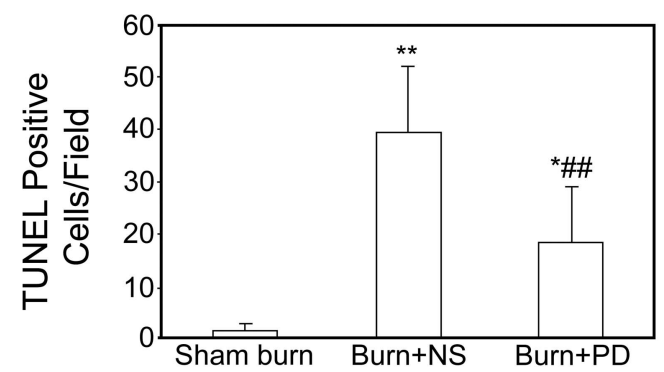

Fig. 7. Representation of terminal deoxyribonucleotidyl transferasemediated deoxyuridine 5-triphosphate-digoxigenin nick end labeling (TUNEL)-positive cells averaged over 10 microscopic fields per animal. Data are presented as the mean \pm SD $(n=6$ in each group). ${ }^{*} P<.05,{ }^{* *} P<.01$ vs the sham burn group; $\# P<.05$; $\# \# P<.01$ vs the burn + normal saline solution (NS) group. Polydatin (PD) treatment improved survival time.

ptosis, which can be significantly alleviated by PD treatment (Fig. 9).

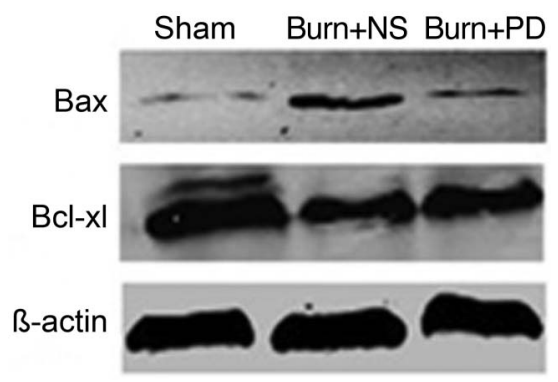

Fig. 8. Expression of $\mathrm{Bcl}-\mathrm{xl}$ and Bax in the lung tissues. The increased Bax expression and decreased $\mathrm{Bcl}-\mathrm{xl}$ expression in the burn + normal saline solution (NS) group was ameliorated by polydatin (PD) treatment.

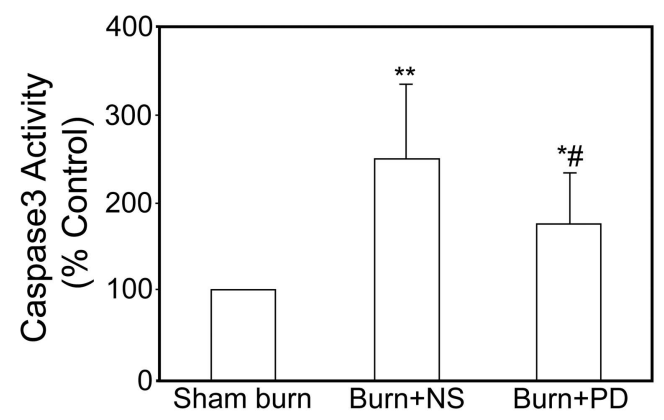

Fig. 9. Caspase-3 activity in the lung tissues. Caspase-3 activity increased in the burn + normal saline solution (NS) group, but it was comparatively decreased in the burn + polydatin (PD) group. Change in caspase- 3 activity is expressed as the percentage of the sham control value. Data are presented as the mean $\pm \mathrm{SD}(n=$ 6 in each group). ${ }^{*} P<.05 ;{ }^{* \star} P<.01$ vs the sham burn group; $\# P<.05$. PD treatment improved survival time.

\section{PD Improves the Survival Time of the Burn-Injured Rats}

To evaluate the protective effect of $\mathrm{PD}$, burn-injury rats were kept in a temperature-controlled room and allowed water ad libitum after PD or NS treatment. As shown in Figure 10 and Table 2, the average survival time extended from $30.2 \pm 11.6 \mathrm{~h}$ in the burn + NS group to $50.7 \pm 17.0 \mathrm{~h}$ in the burn + PD group (sham group vs burn + NS group: $P<.01$; burn + NS group vs burn + PD group: $P=.02$ ). Meanwhile, the 48-h survival rate was 1 of 8 in the burn + NS group, and 4 of 8 in the burn + PD group.

\section{Discussion}

In this study, we were able to show for the first time that PD does indeed confer protection from lung injury in a rat model of burn-induced injury.

Patent lung injury is characterized by the accumulation of a large number of neutrophils, a thickened alveolar wall, and an elevated total number of cells and PMNs in the BALF, and these were all observed in the burn-injury 


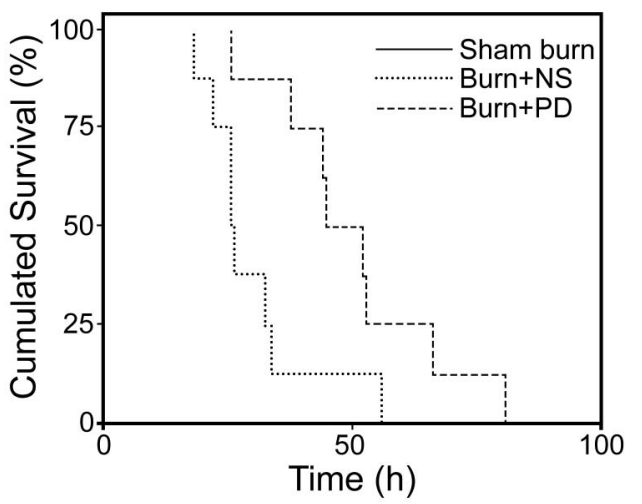

Fig. 10. Survival of treated and untreated rats after burn injury. The survival time of rats improved with polydatin (PD) treatment compared to the burn + normal saline (NS) group.

Table 2. Survival Following Burn Injury (Chi-Square Test)

\begin{tabular}{lccc}
\hline \hline \multicolumn{1}{c}{ Group } & Weight $(\mathrm{g})$ & $\begin{array}{c}\text { Average } \\
\text { survival time }(\mathrm{h})\end{array}$ & $\begin{array}{c}\text { Survival } \\
\text { rate at } 48 \mathrm{~h}\end{array}$ \\
\hline Sham burn & $210.4 \pm 5.6$ & $>96$ & $8 / 8$ \\
Burn + NS & $208.3 \pm 7.7$ & $30.2 \pm 11.6^{*}$ & $1 / 8$ \\
Burn + PD & $210.8 \pm 7.0$ & $50.7 \pm 17.0^{* \dagger}$ & $4 / 8$ \\
& & \\
\multirow{2}{*}{$P<.001$, vs the sham burn group. } & & \\
$\dagger P=.02$, vs. the burn + NS group. & & \\
$\mathrm{NS}=$ normal saline solution \\
$\mathrm{PD}=$ polydatin
\end{tabular}

rats in this study. We found that the administration of PD significantly alleviated these histopathological changes. Moreover, we used increased EB content and W/D as indicators of marked microvascular hyperpermeability and lung edema, respectively, and found that PD was effective in ameliorating both hyperpermeability and edema.

Numerous studies have reported that up-regulation of inflammatory mediators, such as TNF- $\alpha$, IL- $1 \beta$, and IL-6, contributes to the risk of the development of acute SIRS, and the inhibition of these factors prevents the development of this syndrome in burn-injury rats. ${ }^{37,38}$ Our results in the present study indicate that PD can reduce the elevated levels of local and systemic inflammatory mediators induced by burns. It seems reasonable to speculate that this inhibition might be associated with attenuation of pulmonary pathophysiologic alterations caused by severe burn injury. Neutrophilic inflammation is associated with ALI/ acute respiratory distress syndrome. ${ }^{39,40}$ Therefore, we investigated lung neutrophil infiltration by measuring the activity of lung MPO, a neutrophil-specific enzyme. PD treatment prevented the increase in lung MPO activity in burn-injury rats. These results suggest that the administration of PD might exert a protective effect on lung injury induced by burns. Because of the major role of inflamma- tory mediators in lung injury, the effects of PD are probably due to the amelioration of burn-induced lung neutrophil infiltration and inflammation.

Recent studies ${ }^{41}$ have shown that apoptosis contributes to the pathogenesis of lung injury. There is an important theory that links apoptosis to lung injury, according to which apoptosis of alveolar wall cells (ie, epithelial type I and II cells and endothelial cells) as well as the accumulation of neutrophils in the lung might contribute to a cascade of events, and finally to ALI or acute respiratory distress syndrome. ${ }^{20,42}$ Moreover, apoptosis can amplify inflammatory responses in lung diseases. ${ }^{10}$ To determine whether the inhibition of apoptosis was involved in the protective effect of burn-induced lung injury, we examined lung cell apoptosis by TUNEL staining. We found a rather large number of apoptotic cells in the lungs of burninjury animals, and we also found that the number of TUNEL-positive lung cells was significantly reduced after PD treatment. At the molecular level, we investigated several apoptosis-related proteins such as Bcl-xl, Bax, and caspase-3. The $\mathrm{Bcl}$ family consists of both anti-apoptotic (Bcl-2, Bcl-xl) and pro-apoptotic (BAK, BAX) factors. ${ }^{23,43-45}$ The anti-apoptotic members of this family prevent apoptosis by sequestering proforms of death-driving cysteine proteases or by preventing the mitochondrial apoptogenic factors release. On the contrary, the pro-apoptotic members trigger the mitochondrial apoptogenic factors release into the cytoplasm through the mitochondrial permeability transition pore, thereby leading to activation of caspases. Caspase- 3 is considered to be the most important of the executioner caspases and is activated by any of the initiator caspases. ${ }^{46}$ We demonstrated, in this study, that PD treatment prevents burn-induced up-regulation of Bax and caspase- 3 activity and down-regulation of Bcl-xl. These results demonstrated that PD treatment prevents lung cell apoptosis in burn-injury rats.

To further test the protective effect of PD on burn injury, a survival time investigation was performed, and we found that PD administration significantly improved the survival time of burn-injury rats.

We have provided evidence for the first time of PDinduced significant attenuation of lung injury induced by extensive burn injury and improvement in survival time in a rat model. We also found that the potential mechanism of this action is through amelioration of inflammation and apoptosis. Although the involved molecular mechanisms need to be clarified further, this work warrants further investigation of the effect of PD on lung injury and its possible clinical application.

\section{ACKNOWLEDGMENTS}

The authors thank Qiaobing Huang, Professor in Guangdong Key Laboratory of Shock and Microcirculation Research, Department of Patho- 


\section{Polydatin ANd BuRn-INDUCEd Lung InJuRY}

physiology, Southern Medical University, for carefully editing and correcting the manuscript.

\section{REFERENCES}

1. Maybauer MO, Rehberg S, Traber DL, Herndon DN, Maybauer DM. Pathophysiology of acute lung injury in severe burn and smoke inhalation injury. Anaesthesist 2009;58(8):805-812.

2. Rubenfeld GD, Herridge MS. Epidemiology and outcomes of acute lung injury. Chest 2007;131(2):554-562.

3. Turnage RH, Nwariaku F, Murphy J, Schulman C, Wright K, Yin H. Mechanisms of pulmonary microvascular dysfunction during severe burn injury. World J Surg 2002;26(7):848-853.

4. Matthay MA, Ware LB, Zimmerman GA. The acute respiratory distress syndrome. J Clin Invest 2012;122(8):2731-2740.

5. MacCallum NS, Evans TW. Epidemiology of acute lung injury. Curr Opin Crit Care 2005;11(1):43-49.

6. Gokakin AK, Deveci K, Kurt A, Karakus BC, Duger C, Tuzcu M, et al. The protective effects of sildenafil in acute lung injury in a rat model of severe scald burn: a biochemical and histopathological study. Burns 2013;39(6):1193-1199.

7. Zhong WT, Wu YC, Xie XX, Zhou X, Wei MM, Soromou LW, et al. Phillyrin attenuates LPS-induced pulmonary inflammation via suppression of MAPK and NF-kappaB activation in acute lung injury mice. Fitoterapia 2013;90:132-139.

8. Chopra M, Reuben JS, Sharma AC. Acute lung injury:apoptosis and signaling mechanisms. Exp Biol Med (Maywood) 2009;234(4):361371.

9. Jeschke MG, Chinkes DL, Finnerty CC, Kulp G, Suman OE, Norbury WB, et al. Pathophysiologic response to severe burn injury. Ann Surg 2008;248(3):387-401

10. Schmidt EP, Tuder RM. Role of apoptosis in amplifying inflammatory responses in lung diseases. J Cell Death 2010;2010(3):41-53.

11. Bhatia M, Moochhala S. Role of inflammatory mediators in the pathophysiology of acute respiratory distress syndrome. J Pathol 2004;202(2):145-156.

12. Galani V, Tatsaki E, Bai M, Kitsoulis P, Lekka M, Nakos G, et al. The role of apoptosis in the pathophysiology of Acute Respiratory Distress Syndrome (ARDS): an up-to-date cell-specific review. Pathol Res Pract 2010;206(3):145-150.

13. Calfee CS, Eisner MD, Ware LB, Thompson BT, Parsons PE, Wheeler $\mathrm{AP}$, et al. Trauma-associated lung injury differs clinically and biologically from acute lung injury due to other clinical disorders. Crit Care Med 2007;35(10):2243-2250.

14. Lee WL, Downey GP. Neutrophil activation and acute lung injury. Curr Opin Crit Care 2001;7(1):1-7.

15. Huang Q, Xu W, Ustinova E, Wu M, Childs E, Hunter F, et al Myosin light chain kinase-dependent microvascular hyperpermeability in thermal injury. Shock 2003;20(4):363-368.

16. Perl M, Lomas-Neira J, Chung CS, Ayala A. Epithelial cell apoptosis and neutrophil recruitment in acute lung injury-a unifying hypothesis? What we have learned from small interfering RNAs. Mol Med 2008;14(7-8):465-475.

17. Faurschou M, Borregaard N. Neutrophil granules and secretory vesicles in inflammation. Microbes Infect 2003;5(14):1317-1327.

18. Ferkol T, Cohn LA, Phillips TE, Smith A, Davis PB. Targeted delivery of antiprotease to the epithelial surface of human tracheal xenografts. Am J Respir Crit Care Med 2003;167(10):1374-1379.

19. Marko P, Layon AJ, Caruso L, Mozingo DW, Gabrielli A. Burn injuries. Curr Opin Anaesthesiol 2003;16(2):183-191.

20. Kitamura Y, Hashimoto S, Mizuta N, Kobayashi A, Kooguchi K, Fujiwara I, et al. Fas/FasL-dependent apoptosis of alveolar cells after lipopolysaccharide-induced lung injury in mice. Am J Respir Crit Care Med 2001;163(3 Pt 1):762-769.
21. Husari AW, Khayat A, Awdeh H, Hatoum H, Nasser M, Mroueh $\mathrm{SM}$, et al. Activated protein $\mathrm{C}$ attenuates acute lung injury and apoptosis in a hyperoxic animal model. Shock 2010;33(5):467-472.

22. Ware LB, Matthay MA. The acute respiratory distress syndrome. N Engl J Med 2000;342:1334-1349.

23. Li L, Wu W, Huang W, Hu G, Yuan W, Li W. NF-kappaB RNAi decreases the Bax/Bcl-2 ratio and inhibits TNF-alpha-induced apoptosis in human alveolar epithelial cells. Inflamm Res 2013;62(4): 387-397.

24. Childs EW, Tharakan B, Hunter FA, Tinsley JH, Cao X. Apoptotic signaling induces hyperpermeability following hemorrhagic shock. Am J Physiol Heart Circ Physiol 2007;292(6):H3179-H3189.

25. Deng J, Liu W, Wang Y, Dong M, Zheng M, Liu J. Polydatin modulates $\mathrm{Ca}(2+)$ handling, excitation-contraction coupling and beta-adrenergic signaling in rat ventricular myocytes. J Mol Cell Cardiol 2012;53(5):646-656

26. Wang X, Song R, Bian HN, Brunk UT, Zhao M, Zhao KS. Polydatin, a natural polyphenol, protects arterial smooth muscle cells against mitochondrial dysfunction and lysosomal destabilization following hemorrhagic shock. Am J Physiol Regul Integr Comp Physiol 2012; 302(7):R805-R814.

27. Jiang X, Liu W, Deng J, Lan L, Xue X, Zhang C, et al. Polydatin protects cardiac function against burn injury by inhibiting sarcoplasmic reticulum $\mathrm{Ca}$ leak by reducing oxidative modification of ryanodine receptors. Free Radic Biol Med 2013;60C:292-299.

28. Zhao KS, Jin C, Huang X, Liu J, Yan WS, Huang Q, et al. The mechanism of Polydatin in shock treatment. Clin Hemorheol Microcirc 2003;29(3-4):211-217.

29. Lanzilli G, Cottarelli A, Nicotera G, Guida S, Ravagnan G, Fuggetta MP. Anti-inflammatory effect of resveratrol and polydatin by in vitro IL-17 modulation. Inflammation, 2012;35(1):240-248.

30. Cheng Y, Zhang HT, Sun L, Guo S, Ouyang S, Zhang Y, et al. Involvement of cell adhesion molecules in polydatin protection of brain tissues from ischemia-reperfusion injury. Brain Res 2006; 1110(1):193-200.

31. Ravagnan G, De Filippis A, Carteni M, De Maria S, Cozza V, Petrazzuolo M, et al. Polydatin, a natural precursor of resveratrol, induces beta-defensin production and reduces inflammatory response. Inflammation 2013;36(1):26-34.

32. Wang X, Song R, Chen Y, Zhao M, Zhao KS. Polydatin-a new mitochondria protector for acute severe hemorrhagic shock treatment. Expert Opin Investig Drugs 2013;22(2):169-179.

33. Li XH, Gong X, Zhang L, Jiang R, Li HZ, Wu MJ, et al. Protective effects of polydatin on septic lung injury in mice via upregulation of HO-1. Mediators Inflamm 2013;2013:354087.

34. Walker HL, Mason AJ. A standard animal burn. J Trauma 1968; 8(6):1049-1051.

35. Petito CK, Pulsinelli WA, Jacobson G, Plum F. Edema and vascular permeability in cerebral ischemia: comparison between ischemic neuronal damage and infarction. J Neuropathol Exp Neurol 1982;41(4): 423-436.

36. Saria A, Lundberg JM. Evans blue fluorescence: quantitative and morphological evaluation of vascular permeability in animal tissues. J Neurosci Methods 1983;8(1):41-49.

37. Kim YS, Ahn Y, Hong MH, Joo SY, Kim KH, Sohn IS, et al. Curcumin attenuates inflammatory responses of TNF-alpha-stimulated human endothelial cells. J Cardiovasc Pharmacol 2007;50(1):41-49.

38. Qiu X, Li H, Tang H, Jin Y, Li W, Sun Y, et al. Hydrogen inhalation ameliorates lipopolysaccharide-induced acute lung injury in mice. Int Immunopharmacol 2011;11(12):2130-2137.

39. Wheeler AP, Bernard GR. Acute lung injury and the acute respiratory distress syndrome: a clinical review. Lancet, 2007;369(9572): 1553-1564. 


\section{Polydatin ANd BuRn-INDUCEd Lung InJuRY}

40. Matute-Bello G, Frevert CW, Martin TR. Animal models of acute lung injury. Am J Physiol Lung Cell Mol Physiol 2008;295(3):L379L399.

41. Fine A, Janssen-Heininger Y, Soultanakis RP, Swisher SG, Uhal BD. Apoptosis in lung pathophysiology. Am J Physiol Lung Cell Mol Physiol 2000;279(3):L423-L427.

42. Matthay MA. Conference summary: acute lung injury. Chest 1999; 116(1 Suppl):119S-126S

43. Narita M, Shimizu S, Ito T, Chittenden T, Lutz RJ, Matsuda H, Tsujimoto Y. Bax interacts with the permeability transition pore to induce permeability transition and cytochrome $\mathrm{c}$ release in isolated mitochondria. Proc Natl Acad Sci USA 1998;95(25):14681-14686.

44. Du H, Wolf J, Schafer B, Moldoveanu T, Chipuk JE, Kuwana T. BH3 domains other than Bim and Bid can directly activate Bax/Bak. J Biol Chem 2011;286(1):491-501.

45. Kim R, Emi M, Tanabe K. Role of mitochondria as the gardens of cell death. Cancer Chemother Pharmacol 2006;57(5):545-553.

46. Kaufmann SH, Lee SH, Meng XW, Loegering DA, Kottke TJ, Henzing AJ, et al. Apoptosis-associated caspase activation assays. Methods 2008;44(3):262-272. 